\title{
Towards reducing inter- and intra-observer variability: Reasons for optimism?
}

\author{
Brett W. Sperry, MD, a and Wael A. Jaber, MD FACC, FESC ${ }^{\mathrm{b}}$ \\ a Saint Luke's Mid America Heart Institute, Kansas City, MO \\ b Cleveland Clinic Lerner College of Medicine, Fuad Jubran Endowed Chair in Cardiovascular \\ Medicine, Heart and Vascular Institute, Cleveland Clinic, Cleveland, $\mathrm{OH}$
}

Received Oct 12, 2020; accepted Oct 12, 2020

doi: $10.1007 / \mathrm{s} 12350-020-02422-8$

\section{See related article, pp. 440-446}

\begin{abstract}
"Admiration before the general spectacle and skepticism before the individual case." James Baldwin

Cardiac amyloidosis is a disorder where misfolded proteins aggregate as amyloid fibrils into various tissues or organs leading to their dysfunction. The most common type of cardiac amyloidosis was traditionally thought to result from immunoglobulin light chain aggregation (AL amyloidosis). However, with aging of the general population and the re-purposing of bone radiotracers previously used for myocardial infarction imaging and the advent of life-saving medication, there has been a dramatic increase in the diagnosis rates of transthyretin (ATTR) cardiac amyloidosis. Unfortunately, multiple bone radiotracers are available for this indication with availability dependent on the country, and there are multiple imaging protocols and time points at which to acquire images. This has made standardization and interpretation of sensitivity, specificity, and diagnostic accuracy of various reports from different centers and countries challenging. Practice points put out by ASNC and a multisociety expert consensus recommendations document have attempted to provide clarity to the indications for imaging, protocols, and interpretations using these radiotracers. ${ }^{1,2}$ However, multiple imaging protocols are suggested and
\end{abstract}

\footnotetext{
Reprint requests: Wael A. Jaber, MD FACC, FESC, Cleveland Clinic Lerner College of Medicine, Fuad Jubran Endowed Chair in Cardiovascular Medicine, Heart and Vascular Institute, Cleveland Clinic, 9500 Euclid Ave. J1-5, Cleveland, OH 44195; JABERW@ccf.org

J Nucl Cardiol 2022;29:447-8.

1071-3581/\$34.00

Copyright (C) 2020 American Society of Nuclear Cardiology.
}

comparison among these protocols as well as inter- and intra-observer variability analyses are limited.

The current study in this issue of the Journal of Nuclear Cardiology ${ }^{\circledR}$ by Singh et al. evaluated 100 consecutive patients at the Brigham and Women's Hospital who were referred for technetium pyrophosphate (TcPYP) for the diagnosis of transthyretin cardiac amyloidosis (ATTR). Both planar and SPECT/CT images were obtained and were analyzed by the original reading physician, an additional experienced nuclear imager, and 2 novice nuclear imagers to assess inter- and intra-observer variability. About half of the images were acquired after 1-hour incubation and the rest at 2.5-3hour incubation. The results of the study showed a very high correlation for $\mathrm{H} / \mathrm{CL}$ ratio on the planar images which is reassuring and has been described in several prior studies. $^{3,4}$ Analysis of the SPECT/CT images showed a $100 \%$ correlation with respect to the study being considered positive or negative for ATTR among both the experienced and novice readers. The authors conclude that there is excellent inter- and intra-observer repeatability of TcPYP visual interpretation with SPECT/CT and H/CL ratio, and that ATTR cardiac amyloidosis can be diagnosed reliably using these imaging protocols.

As TcPYP nuclear imaging is expanding outside of academic referral centers to more novice readers, this manuscript is timely and important for the nuclear community. One important caveat is that a minority of nuclear labs, particularly outside of academic centers, have SPECT/CT cameras. Additionally, even the novice readers in this study were training at a high-volume amyloidosis center and likely have more exposure to this imaging modality than many nuclear readers starting to use this technology in the community. It is reassuring that there was $100 \%$ concordance at studies acquired both at the 1- and 3-hour incubation time points. It currently remains unclear which is the ideal time point 
for imaging. However, several recent studies have shown identical findings during blinded reads in patients who were imaged at both time points, suggesting that these shorter 1-hour protocol may be optimal, particularly when SPECT/CT is used. ${ }^{45}$ The longer 3 -hour study should be considered when there is persistent blood pool, particularly when CT attenuation correction is not available. ${ }^{2}$

The challenges ahead are thrilling and humbling. This report and many others are derived from centers where patients presented with symptoms of heart failure or other markers of clinical involvement with ATTR by ECG and/or echocardiography and therefore high pretest likelihood of disease with advanced disease stage. It is important to question the diagnostic performance of our scintigraphy imaging for ATTR once we start testing subjects in the pre-symptomatic stage based on family history or genetic markers. The cautionary road ahead for bone scintigraphy is long but nonetheless essential to maintain the current trust in its diagnostic performance:

(1) What is the positive diagnostic yield of TcPYP at major centers and is it falling?

(2) How early in the disease does TcPYP imaging converts to "positive"?

(3) Does TcPYP have similar diagnostic performance in all genetic variants of ATTR?

(4) In asymptomatic patients at risk, is repeat scanning needed and when and what is the threshold for converting from a negative scan to a positive scan?

(5) Is the interobserver variability similar in patients with ratios between $\mathrm{H} / \mathrm{CL}$ of 1.3 and 1.5?

(6) Can TcPYP track the clinical improvement derived from new therapies on repeat scanning?
As seen in this report, the diagnostic performance of this old tracer in large groups of patient with ATTR and in our modern era deserves great admiration. For the future to remain bright, TcPYP performance should be challenged to deliver similar results for individual patients at the margins.

\section{Disclosure}

Authors declare that they have no conflicts of interest to disclose.

\section{References}

1. Dorbala S, Bokhari S, Miller EJ, Bullock-Palmer R, Soman P, Thompson R. ${ }^{99 \mathrm{~m}}$ Technetium-pyrophosphate imaging for transthyretin cardiac amyloidosis. ASNC practice points. https://www.asnc. org/Files/Practice\%20Resources/Practice\%20Points/ASNC\%20Pra ctice\%20Point-99mTechnetiumPyrophosphateImaging2016.pdf.

2. Dorbala S, Ando Y, Bokhari S, et al. ASNC/AHA/ASE/EANM/ HFSA/ISA/SCMR/SNMMI expert consensus recommendations for multimodality imaging in cardiac amyloidosis: Part 1 of 2-evidence base and standardized methods of imaging. J Nucl Cardiol 2019;26:2065-123.

3. Bokhari S, Morgenstern R, Weinberg R, et al. Standardization of 99mTechnetium pyrophosphate imaging methodology to diagnose TTR cardiac amyloidosis. J Nucl Cardiol 2018;25:181-90.

4. Sperry BW, Burgett E, Bybee KA, et al. Technetium pyrophosphate nuclear scintigraphy for cardiac amyloidosis: Imaging at 1 vs 3 hours and planar vs SPECT/CT. J Nucl Cardiol Off Publ Am Soc Nucl Cardiol 2020;27:1802-7.

5. Masri A, Bukhari S, Ahmad S, et al. Efficient 1-Hour Technetium$99 \mathrm{~m}$ Pyrophosphate Imaging Protocol for the Diagnosis of Transthyretin Cardiac Amyloidosis. Circ Cardiovasc Imaging 2020;13:e010249.

Publisher's Note Springer Nature remains neutral with regard to jurisdictional claims in published maps and institutional affiliations. 